\title{
First principles calculation of lithium-phosphorus co-doped diamond
}

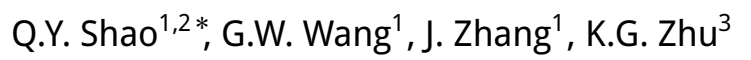 \\ ${ }^{1}$ Laboratory of Quantum Information Technology, School of Physics and Telecommunication Engineering, \\ South China Normal University, Guangzhou 510006, China \\ 2 Department of Physics, Zhangzhou Normal University, Zhangzhou 363000, China \\ 3 Department of Physics, Beihang University, Beijing 100191, China
}

Received November 27, 2011, in final form June 30, 2012

\begin{abstract}
We calculate the density of states (DOS) and the Mulliken population of the diamond and the co-doped diamonds with different concentrations of lithium (Li) and phosphorus (P) by the method of the density functional theory, and analyze the bonding situations of the Li-P co-doped diamond thin films and the impacts of the Li-P co-doping on the diamond conductivities. The results show that the Li-P atoms can promote the split of the diamond energy band near the Fermi level, and improve the electron conductivities of the Li-P co-doped diamond thin films, or even make the Li-P co-doped diamond from semiconductor to conductor. The effect of Li-P co-doping concentration on the orbital charge distributions, bond lengths and bond populations is analyzed. The Li atom may promote the split of the energy band near the Fermi level as well as may favorably regulate the diamond lattice distortion and expansion caused by the $\mathrm{P}$ atom.
\end{abstract}

Key words: Li-P co-doped diamond, density of states, impurity level, orbital charge

PACS: 71.15.Mb, 71.20.-b, 81.05.Uw, 71.55.Cn

\section{Introduction}

Diamond has a great potential for applications. It has a wide band gap, high breakdown voltage, high carrier mobility, high thermal conductivity and chemical inertness and so on. By incorporating the boron atoms into a diamond, the diamond thin films can acquire shallow acceptor impurity levels near the top of its valence band, and become the $p$-type semiconductors. Recently, the $p$-type diamonds have received a lot of developments, and have had a wide range of applications in the industry, such as the applications of electrodes [1], the detection applications [2, 3] and the preparations of semiconductor devices [4], and so on. In the last two years, the superconducting characteristics of the boron-doped diamond thin films have attracted a great deal of attention [5-7]. However, the preparations of the N-type diamond thin films did not have much progress, which greatly restricted the developments of the diamond semiconductor devices. N-type impurity atoms are mainly lithium atoms and the sodium atoms in group I of the Periodic Table, the nitrogen atoms and the phosphorus atoms in group V, and the sulfur atoms and the oxygen atoms in group VI. In 1996, by theoretical calculations, R. Jones found that the phosphorus vacancy complexes were deep acceptors, and were capable of compensating any donor and making the phosphorus-doped diamond remain an insulator [8]. In 2005, the thermal ionization energy and the capture cross-section of the phosphorus donor were estimated to $0.54 \pm 0.02 \mathrm{eV}$ and $(4.5 \pm 2.0) \times 10^{-17} \mathrm{~cm}^{2}$ in the experiment which analyzed the conductivity of a phosphorus-doped diamond [9]. The electrical properties and the shapes of phosphorus-doped diamonds, which are prepared by an organic phosphorus gas $\left[\mathrm{P}\left(\mathrm{C}_{4} \mathrm{H}_{9}\right) \mathrm{H}_{2}\right.$ and $\left.\mathrm{P}\left(\mathrm{CH}_{3}\right)_{3}\right]$ or by an inorganic phosphorus gas $\mathrm{PH}_{3}$, are the same in the experiment of the preparation of a phosphorus-doped diamond by the plasma enhanced chemical vapor deposition

\footnotetext{
*E-mail: qyshao@163.com, Phone: +86-20-39310066, Fax: +86-20-39310882
} 
method [10]. At the same time, in the analysis of the relationship between the mobility of the phosphorusdoped diamond's (001) surface and temperature, the largest mobility (approximately $450 \mathrm{~cm}^{2} / \mathrm{Vs}$ ) lies in $260 \mathrm{~K}$, and the mobility at room temperature is $350 \mathrm{~cm}^{2} / \mathrm{Vs}$ [11]. In 2006, when Takatoshi Yamada and his collaborators studied the field emission properties of heavy phosphorus-doped diamond thin films, they found that the reconstruction film surface which was annealed in vacuum, had a minimum threshold field value $16 \mathrm{~V} / \mu \mathrm{m}$, while the threshold field values of the thin film surface which was terminated by oxygen or hydrogen were $28 \mathrm{~V} / \mu \mathrm{m}$ or $44 \mathrm{~V} / \mu \mathrm{m}$ [12]. In 2008, J. Pernot and his collaborators found that at the phosphorus atom concentrations being less than $10^{17} \mathrm{~cm}^{-3}$, the electron mobility was determined by the lattice scattering; when the phosphorus atom concentrations were between $10^{17} \mathrm{~cm}^{-3}$ and $10^{18} \mathrm{~cm}^{-3}$, the electron mobility was determined by both the lattice scattering and by the scattering of ionized impurity atoms; when the phosphorus atom concentrations were higher than $10^{18} \mathrm{~cm}^{-3}$, the electron mobility was determined by the scattering of neutral impurity atoms [13]. Although both the experiments and theories of only single phosphorus atoms doped diamond have greatly increased and improved, the electron conductivities of the phosphorus-doped diamond thin films remain low in experiments and cannot meet the requirements for the preparations of semiconductor devices. Thus, some researchers referred to the doping experiments of the GaAs and advised that the co-doping method might be a good way. In 1995, the experiment of the preparation of the nitrogen-phosphorus co-doped diamond thin films by hotfilament chemical vapor deposition showed that adding some nitrogen atoms was advantageous to the phosphorus-doping and to the increase of the growth rate of the films, as well as can get a higher doping concentration: the maximum concentration of the phosphorus atoms and the nitrogen atoms were $3 \times 10^{19}$ and $6 \times 10^{19}$ atoms $/ \mathrm{cm}^{3}$, respectively [14]. In 2004, when Li Rong-Bin and his collaborators prepared the boron-sulfur co-doped diamond thin films by traditional microwave plasma chemical vapor deposition, they found that the addition of some boron atoms could be advantageous to sulfur doping, and made the amount of the sulfur atoms increase 1.5 times while the activation energy of the electron conductivity was reduced from $0.52 \mathrm{eV}$ to $0.39 \mathrm{eV}$ [15]. In 2005, W.S. Lee and his collaborators found that the Hall coefficient of the boron-lithium co-doped diamond thin films was $-2.974 \times 10^{-2} \mathrm{~cm}^{3} / \mathrm{C}$, and its resistivity was $0.01 \div 0.02 \Omega \mathrm{m}$. They also confirmed that the co-doping method could improve the stability of the lithium in the diamond thin films [16]. In 2007, E.B. Lombardi and his collaborators also studied the interstitial doping and the substitution doping of the lithium and sodium atoms. By the experiment they confirmed that the lithium atom was an interstitial atom and the sodium atom was a substitution atom [17]. In 2009, according to the calculation of the first principle, F. Iori found that the co-doped diamond, where two kinds of impurity atoms lied in the nearest neighbor, has the lowest impurity formation energy [18].

In recent years, although the co-doping method had been tried a lot in experiments and theories, it did not get much progress. The studies of the Li-P co-doped diamonds are very few. Therefore, in this paper, based on the first principle of the density functional theory (DFT), we calculate the Mulliken population and the DOS of the co-doped diamonds with different concentrations of Li and P, analyze their electronic structures, and determine the bonding properties and the charge distributions among lithium atoms, phosphorus atoms and carbon atoms and the impacts on the electrical properties after doping.

\section{Calculation method}

In this paper, we do our calculation work with $a b$ initio calculation quantum mechanics module Cambridge Serial Total Energy Package (CASTEP) which is based on the density functional theory in Accelrys Materials Studio software [19]. The module uses local atom-based groups and the numerical periodic boundary condition to describe the valence electrons while the interactions between electrons and ions is mainly described by the Norm-Conserving Pseudopotential and the Ultrasoft Pseudopotential [20]. In order to minimize the number of plane wave basis sets, we chose the Ultrasoft Pseudopotential to describe the interactions between electrons and ions, while the valence electron configurations of the carbon atom, the phosphorus atom, the lithium atom are respectively selected to be C: $2 s^{2} 2 p^{2}$, P: $3 s^{2} 3 p^{3}$ and Li: $1 s^{2} 2 s^{1}$. The cut off energy of the plane waves is $300.0 \mathrm{eV}$, and the exchange-correlation energy is described by the PBE parameterized form of the generalized gradient approximation (GGA) [21]. In this paper, we only calculate the situation of two kinds of impurity atoms lying in the nearest neighbor. In 


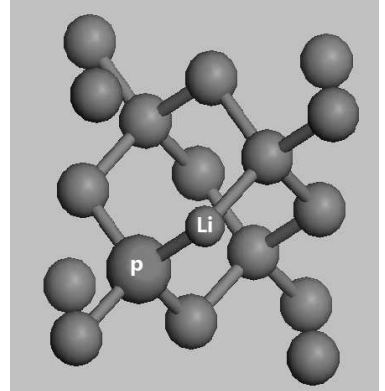

(a)

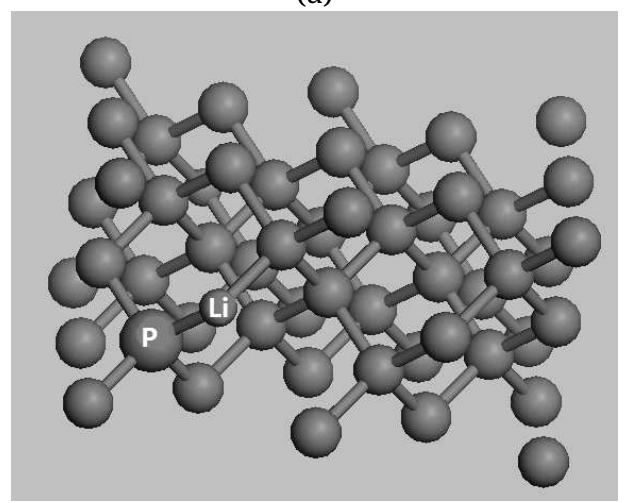

(c)

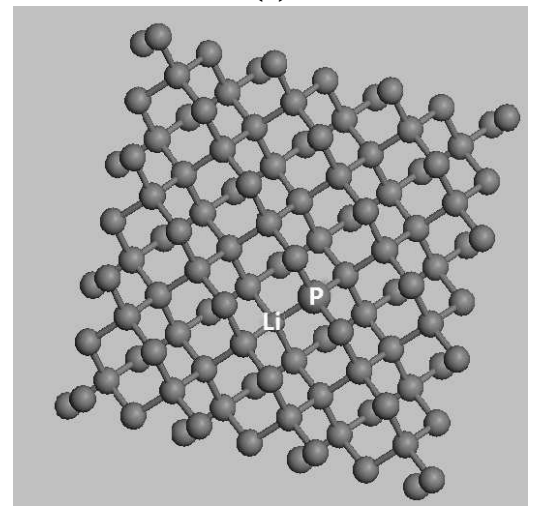

(e)

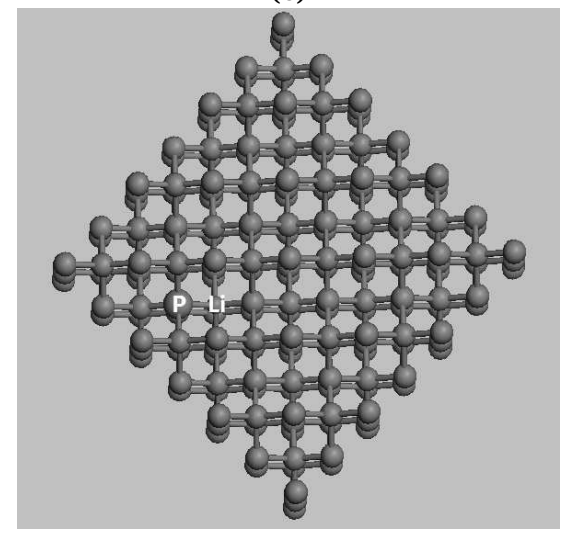

(g)

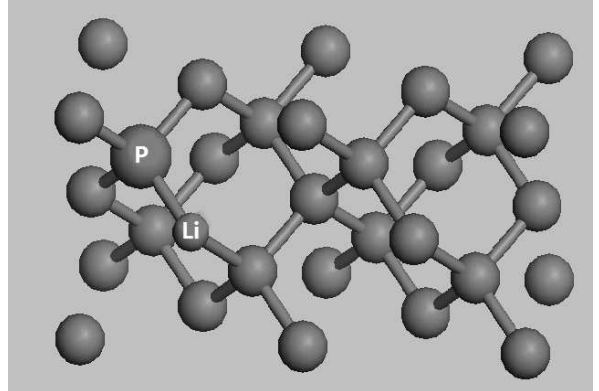

(b)

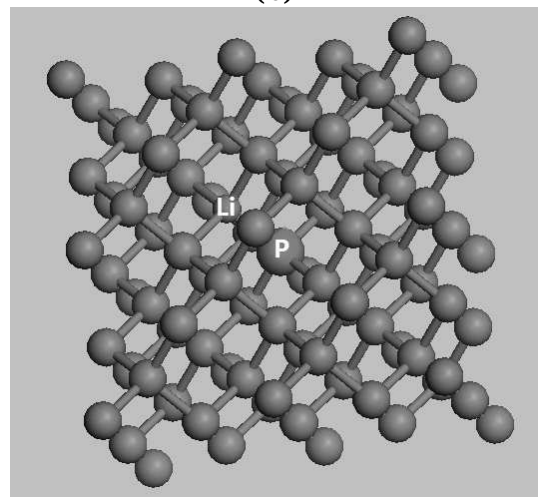

(d)

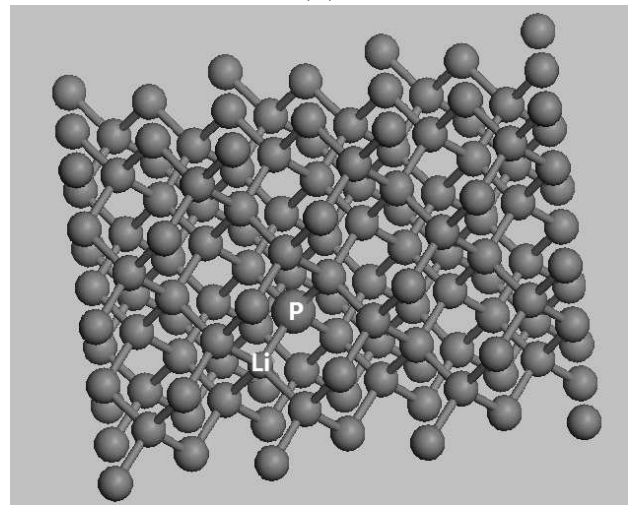

(f)

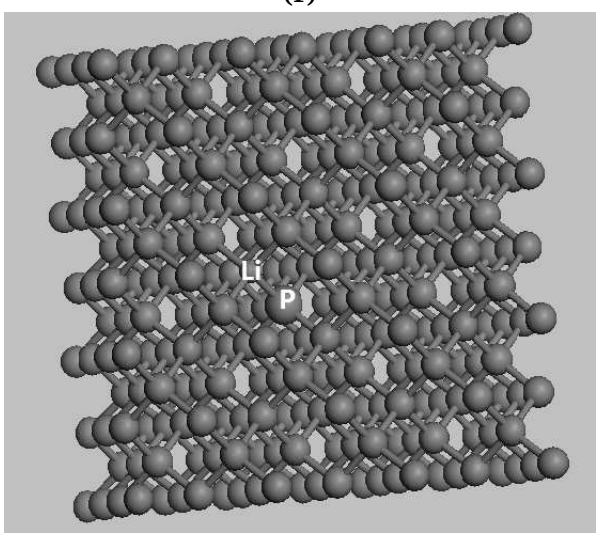

(h)

Figure 1. The structure of the doped diamond where the number of Li atom is one, $\mathrm{P}$ atom's number is one and the C atom's number is (a) six, (b) fourteen, (c) thirty, (d) sixty-two, (e) seventy, (f) ninety-four, (g) one-hundred-forty-two, (h) two-hundred-fourteen. 
this way, a Li atom and a phosphorus atom are incorporated into the diamond lattice with an atom pair at a random site. In order to study the Li-P co-doped diamond lattices of different concentrations, we separately calculate the diamond lattices where the ratio of the lithium atom, phosphorus atom, and the carbon atoms are $1: 1: 6,1: 1: 14,1: 1: 30,1: 1: 62,1: 1: 70,1: 1: 94,1: 1: 142,1: 1: 214$, and the structures are shown in figure 1 . Their $k$ values are set for $7 \times 7 \times 7,4 \times 7 \times 7,4 \times 4 \times 7,4 \times 4 \times 4,2 \times 2 \times 7$, $2 \times 4 \times 4,2 \times 2 \times 4,2 \times 2 \times 2$, in order to ensure the convergences of the system's energy and the structures in the plane-wave basis set.

In the SCF calculation, we chose the Pulay density hybrid approach, and set the SCF to $1.0 \times$ $10^{-6} \mathrm{eV} \cdot$ atom $^{-1}$. In the geometric optimization, we chose the BFGS algorithm. The accuracy of total energy was $1.0 \times 10^{-5} \mathrm{eV} \cdot$ atom $^{-1}$, the crystal force of each atom was less than $0.3 \mathrm{eV} \cdot \mathrm{nm}^{-1}$, the stress of each structural unit was less than $0.05 \mathrm{GPa}$, and the atomic displacement caused by the changes of the structural parameters was less than $1.0 \times 10^{-4} \mathrm{~nm}$.

\section{Calculation results and discussions}

\subsection{Analysis of electron density of states of the lithium-phosphorus co-doped diamond}

In this paper, we calculated the DOS of the diamond and the co-doped diamonds with different concentrations of $\mathrm{Li}$ and $\mathrm{P}$. The total density of states (TDOS) of the diamond and the partial density of states (PDOS) of the diamond are showed in figure 2, The middle point line is the Fermi level. Its left is the valence band and its right is the energy gap and the conduction band. The energy gap is $4.138 \mathrm{eV}$ and it was a little different from the value of the experimental measurement $5.4 \mathrm{eV}$. The phenomenon of the energy gap being underestimated lies prevalently in the density functional calculations [22], but it does not affect our following qualitative analysis on the doped diamonds. The valence band of a diamond consists of two areas: in the high energy region (approximately $-13 \div 0 \mathrm{eV}$ ), it is mainly occupied by the $\mathrm{C} 2 p$ states, while in the low energy region (approximately $-21.5 \div-13 \mathrm{eV}$ ), it is mainly occupied by the $\mathrm{C} 2 s$ states, and the conduction band of the diamond is mainly occupied by the $\mathrm{C} 2 p$ states. From the PDOS in the conduction band, we can see that the area ratio of the $\mathrm{C} 2 s$ states and $\mathrm{C} 2 p$ states is approximately $1: 3$. Here, although the above result has been known for a long time, we illustrate it again in order to be compared with the situations of a doped diamond.

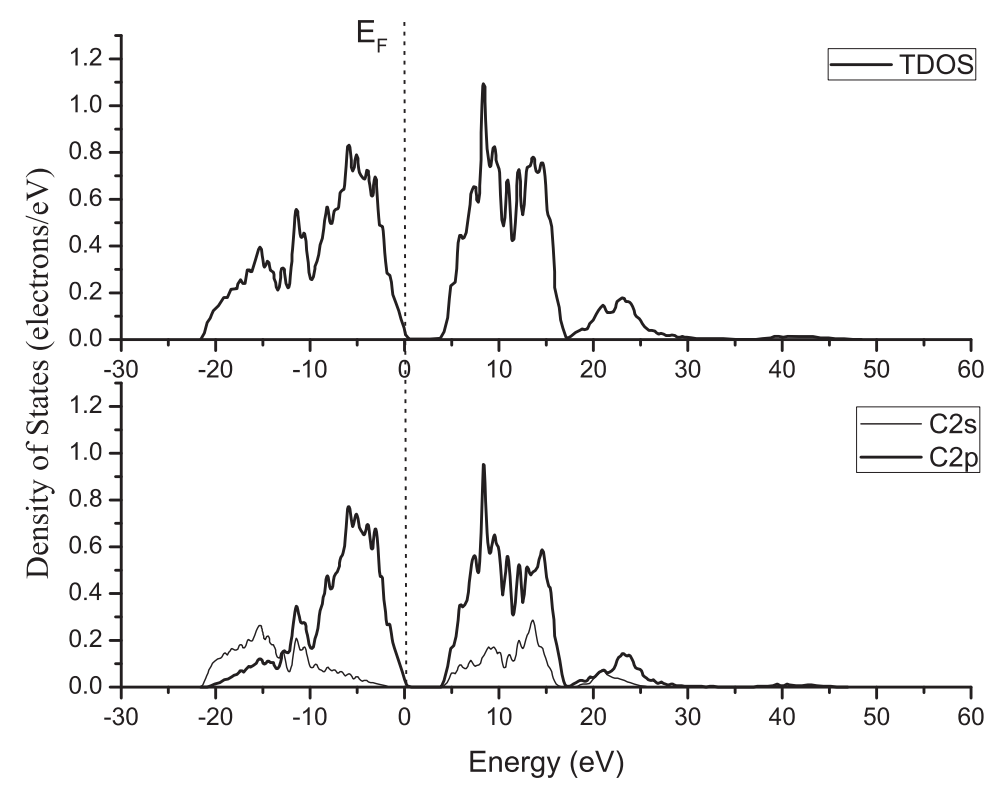

Figure 2. The total density of states (TDOS) and the partial density of states of the diamond. The $E_{\mathrm{F}}$ stands for the Fermi level. The C $2 s$ and $\mathrm{C} 2 p$ represent the electric charge distributions of $2 s$ and $2 p$ orbits. 

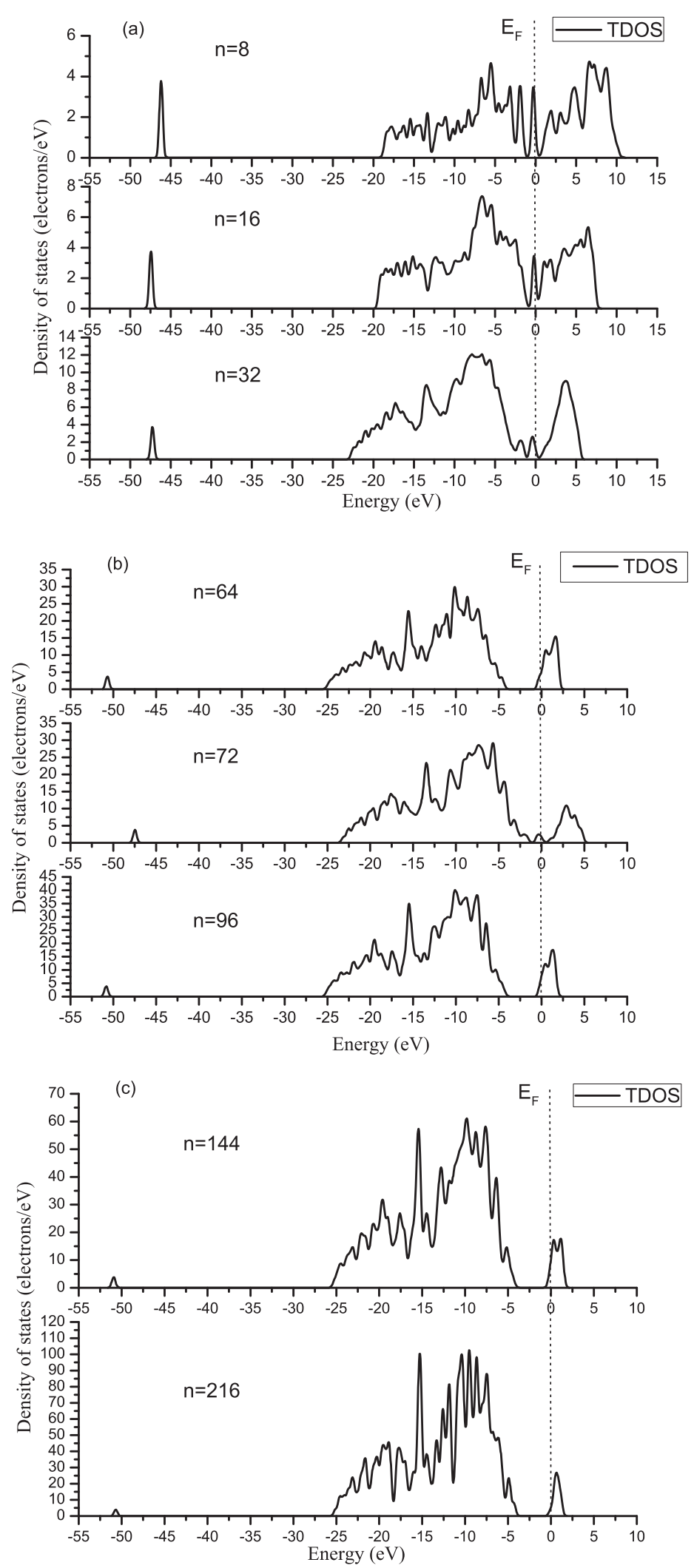

Figure 3. The total density of states (TDOS) of the Li-P co-doped diamond, $n$ is the total atomic number, and each cell has a lithium atom and a phosphorus atom (the Li-P atom-pair). The $E_{\mathrm{F}}$ stands for the Fermi level. 
Comparing the TDOS of the Li-P co-doped diamond in figure 3 with the TDOS of the diamond in figure 2 we can see that the Fermi level of a diamond after doping obviously moves near the bottom of conduction band. When the doping concentration is relatively low, such as the TDOS shown in figure 3(b) and (c), the Fermi level of the Li-P co-doped diamond moving near the bottom of the conduction band, their energy gap width is almost the same as the diamond's (as shown in figure 2), and the donor levels are formed in the band gap and near the bottom of the conduction band. However, when the doping concentration is higher, such as the TDOS shown in figure 3(a), the energy gap of the Li-P co-doped diamond disappears. This is most probably caused by the impurity level broadening and an impurity band formation, which completely fills the energy gap region. Thus, the Li-P co-doped diamond becomes a conductor. All this illustrates that when the donor impurity atoms are incorporated into a diamond, the impurity levels will appear near the bottom of the conduction band, and then the insulated diamond will become a semiconductor which has some conductivity. When the concentrations of the lithium atom and phosphorus atom are not too high, only some impurity levels are formed near the bottom of the conduction band. But when the concentrations of the lithium atom and phosphorus atom are high enough, the impurity levels will increase, broaden and become an impurity band, or even extend to the entire energy gap. Then, they will make the energy gap disappear, and the semiconductor will become a conductor. Here, the magnitude of concentration of the lithium atom or the phosphorus atom is about $10^{21}$, three orders of magnitude higher than the concentration of the phosphorus atoms in the reference [10] where its concentration is about $(2 \div 3) \times 10^{18} \mathrm{~cm}^{-3}$, and two orders of magnitude higher than the concentration of the phosphorus atoms in the reference [12] where its concentration is determined to be $7 \times 10^{19} \mathrm{~cm}^{-3}$. Although some impurity atom concentrations of the calculation models in this paper are higher than in the experiment, the change or tendency of the doped diamond for different doping concentrations in this paper is clear, so it does not affect our analysis. In addition, we also found that in the TDOS of the doped diamond whose energy gap has disappeared (as shown in figure 3), the peak value of the DOS of the valence band of a lithium atom lies between about $-48.5 \div-45 \mathrm{eV}$, while in the TDOS of the doped diamond whose energy gap has not disappeared, the peak value of the DOS of the valence band of a lithium atom lies between about $-51.5 \div-50 \mathrm{eV}$. This indicates that when the doping concentration of the Li-P co-doped diamond is high, the DOS of the valence band of a lithium atom will move toward the high energy direction. In short, when the atomic ratio of the lithium atoms or the phosphorus atoms to

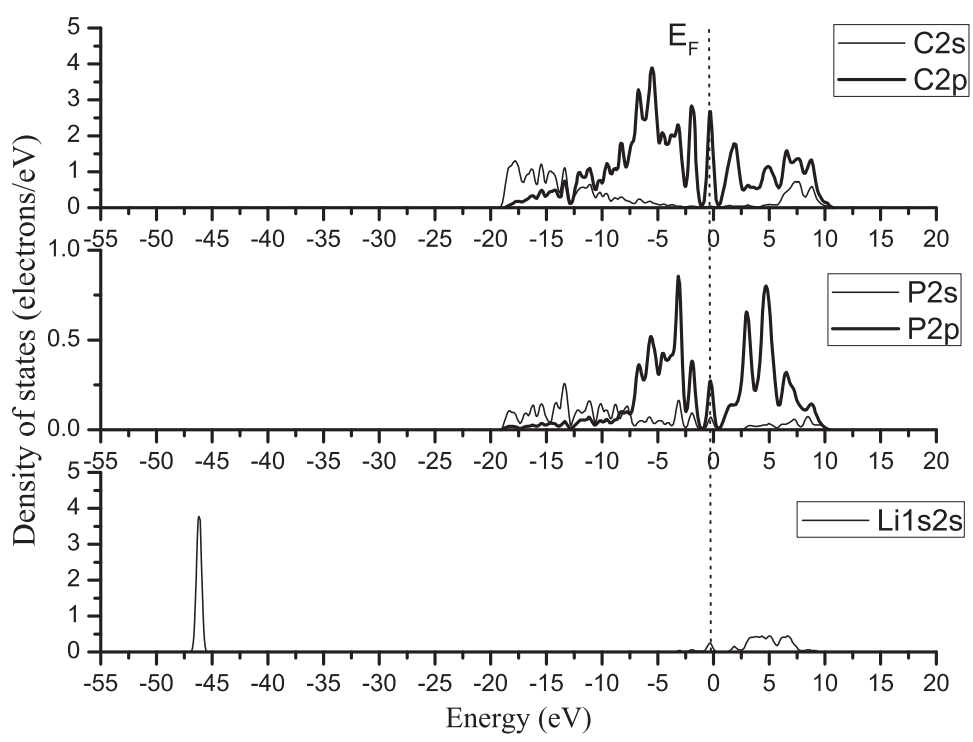

Figure 4. The partial density of states of the doped diamond where the number of Li atom is one, $\mathrm{P}$ atom's number is one and the C atom's number is six. The $E_{\mathrm{F}}$ stands for the Fermi level. The C2s and $\mathrm{C} 2 p$ represent the electric charge distributions of $2 s$ and $2 p$ orbits of the carbon atoms. The P2s and $\mathrm{P} 2 p$ represent the electric charge distributions of $2 s$ and $2 p$ orbits of the phosphorus atoms. The Lil $s 2 s$ represent the electric charge distributions of $1 s$ and $2 s$ orbits of the lithium atoms. 


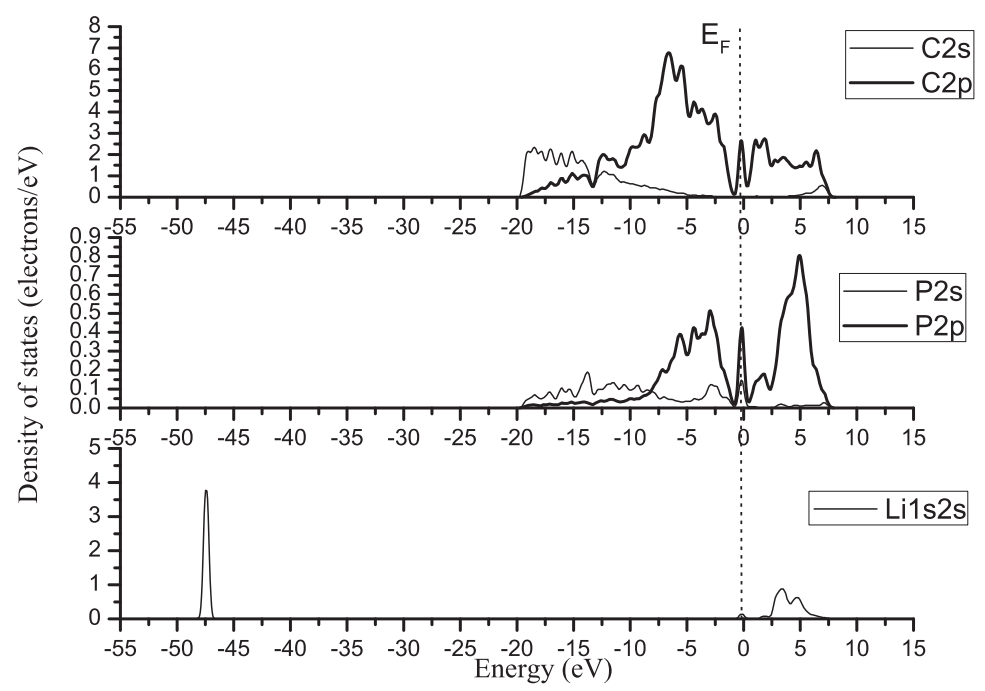

Figure 5. The partial density of states of the doped diamond where the number of Li atom is one, P atom's number is one and the $\mathrm{C}$ atom's number is fourteen. The $E_{\mathrm{F}}$ stands for the Fermi level. The $\mathrm{C} 2 s$ and $\mathrm{C} 2 p$ represent the electric charge distributions of $2 s$ and $2 p$ orbits of the carbon atoms. The P $2 s$ and $\mathrm{P} 2 p$ represent the electric charge distributions of $2 s$ and $2 p$ orbits of the phosphorus atoms. The Li1 $s 2 s$ represent the electric charge distributions of $1 s$ and $2 s$ orbits of the lithium atoms.

the carbon atoms is less than $1: 70$ (or the concentration of the lithium atom or the phosphorus atom is less than $2.35 \times 10^{21} \mathrm{~cm}^{-3}$ ), the Li-P co-doped diamonds are always semiconductors, otherwise they are conductors.

In order to further analyze the impacts of the Li-P atom-pairs co-doping on the electrical properties of the diamond, we also calculated the PDOS of the Li-P co-doped diamond of different doping concentrations (as shown in the figure 4 11). From these pictures, we can see that when the doping concentration is high (as shown in figures 4 and 5), the $1 s$ and $2 s$ electrons of the lithium atom have some contribu-

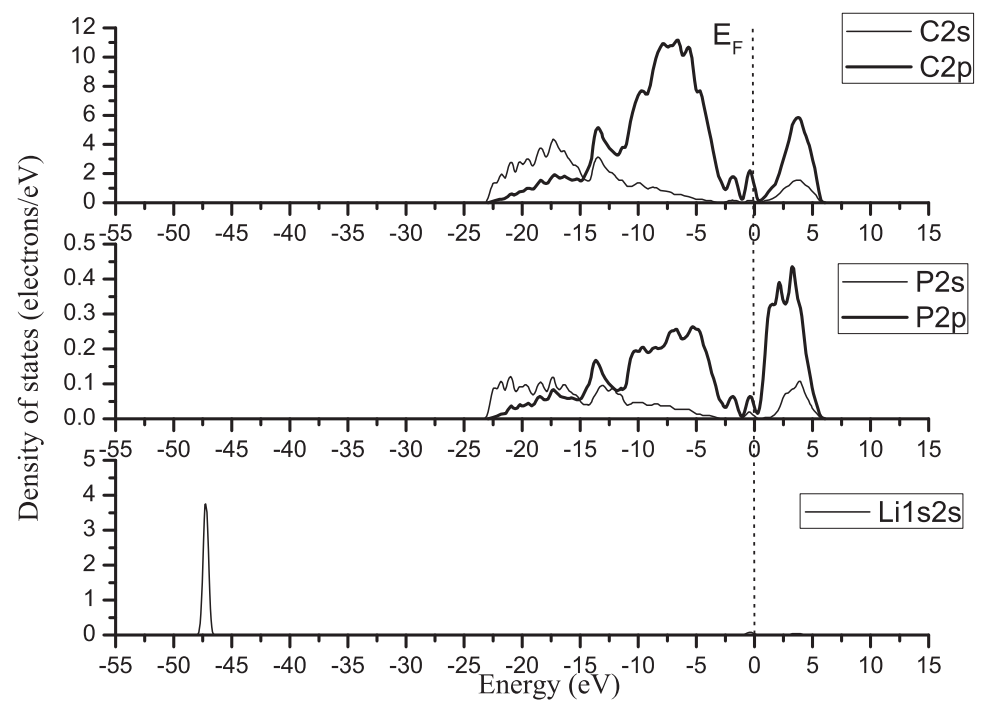

Figure 6. The partial density of states of the doped diamond where the number of Li atom is one, $\mathrm{P}$ atom's number is one and the C atom's number is thirty. The $E_{\mathrm{F}}$ stands for the Fermi level. The C2s and $\mathrm{C} 2 p$ represent the electric charge distributions of $2 s$ and $2 p$ orbits of the carbon atoms. The $\mathrm{P} 2 s$ and P2 $p$ represent the electric charge distributions of $2 s$ and $2 p$ orbits of the phosphorus atoms. The Lil $2 s$ represent the electric charge distributions of $1 s$ and $2 s$ orbits of the lithium atoms. 


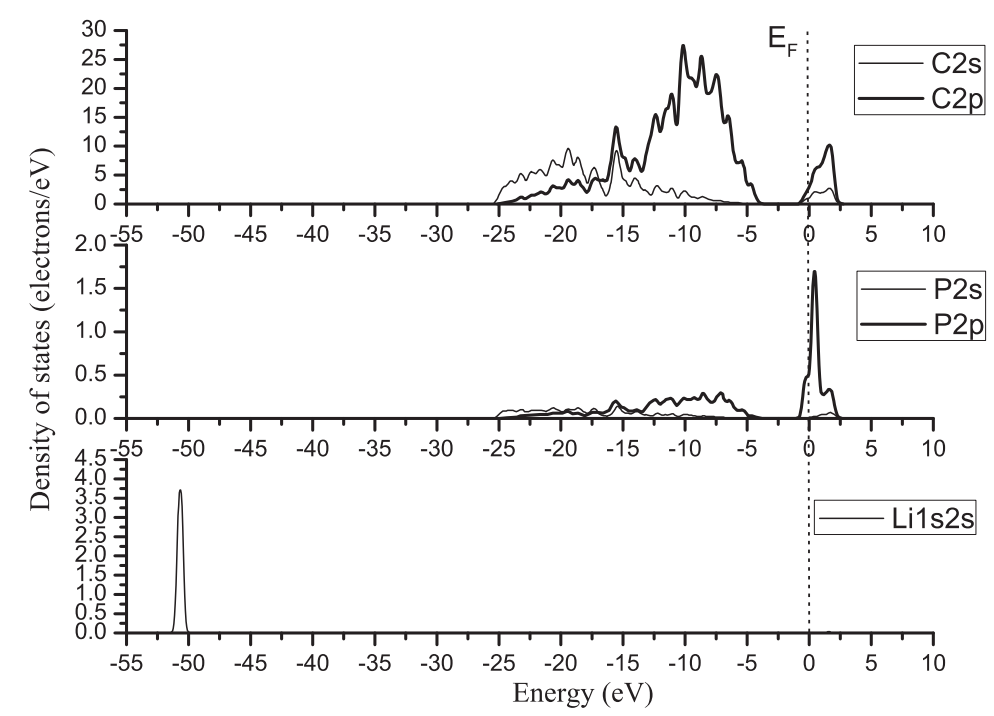

Figure 7. The partial density of states of the doped diamond where the number of Li atom is one, $\mathrm{P}$ atom's number is one and the $\mathrm{C}$ atom's number is sixty-two. The $E_{\mathrm{F}}$ stands for the Fermi level. The $\mathrm{C} 2 s$ and $\mathrm{C} 2 p$ represent the electric charge distributions of $2 s$ and $2 p$ orbits of the carbon atoms. The $\mathrm{P} 2 s$ and P2 $p$ represent the electric charge distributions of $2 s$ and $2 p$ orbits of the phosphorus atoms. The Lil $s 2 s$ represent the electric charge distributions of $1 s$ and $2 s$ orbits of the lithium atoms.

tions to the conduction band near the Fermi level; and when the doping concentration is low (as shown in figure 6 [11), the $s$ electrons of the lithium atom nearly have no contribution to the conduction band near the Fermi level. Therefore, on the one hand, we can improve the electron conductivity of the heavy phosphorus-doped diamond by incorporating some lithium atoms; on the other hand, when the lithium atoms are incorporated into the non-heavy phosphorus-doped diamond, although the lithium atoms almost have no impacts on the conductive properties of the phosphorus-doped diamond, they can effec-

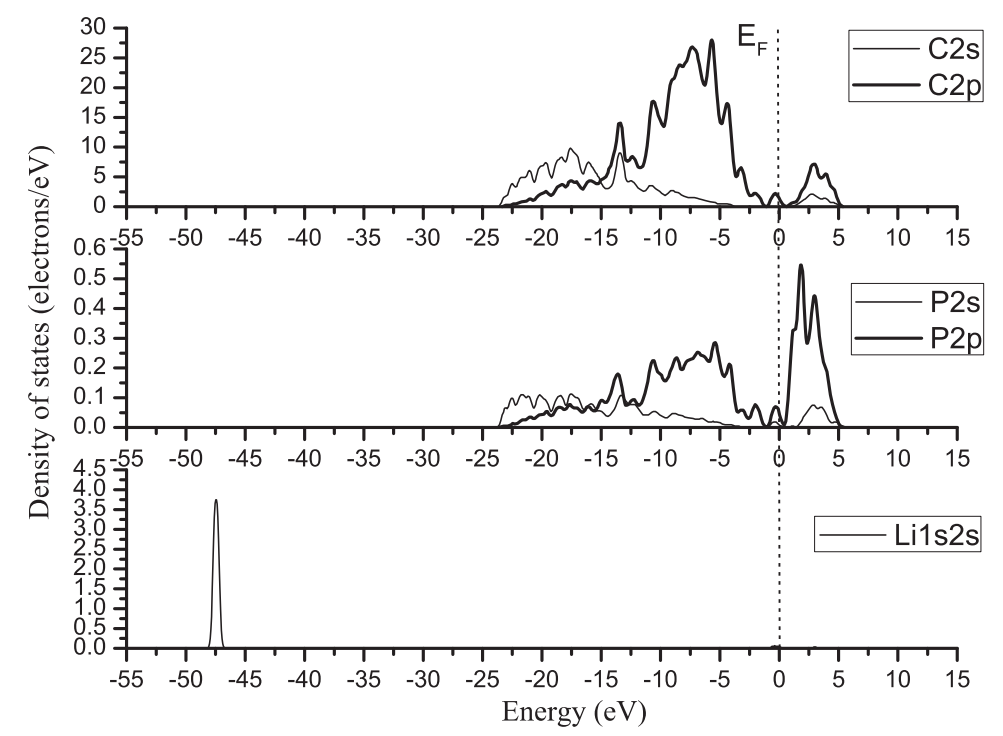

Figure 8. The partial density of states of the doped diamond where the number of Li atom is one, $\mathrm{P}$ atom's number is one and the C atom's number is seventy. The $E_{\mathrm{F}}$ stands for the Fermi level. The C2s and $22 p$ represent the electric charge distributions of $2 s$ and $2 p$ orbits of the carbon atoms. The P2s and P2 $p$ represent the electric charge distributions of $2 s$ and $2 p$ orbits of the phosphorus atoms. The Lil $s s$ represent the electric charge distributions of $1 s$ and $2 s$ orbits of the lithium atoms. 


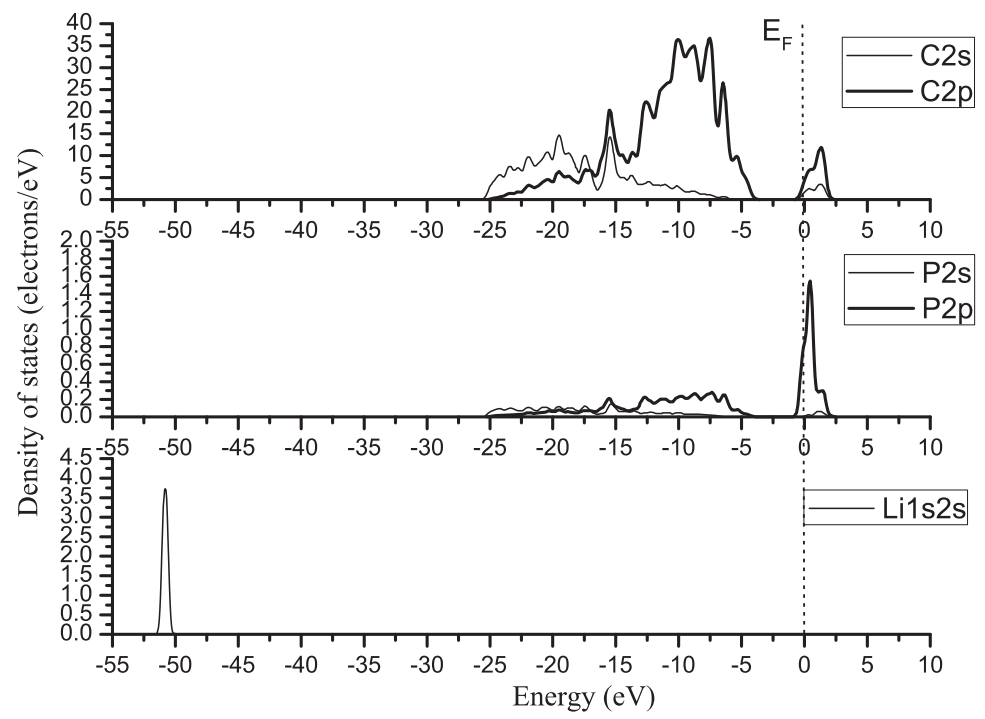

Figure 9. The partial density of states of the doped diamond where the number of $\mathrm{Li}$ atom is one, $\mathrm{P}$ atom's number is one and the C atom's number is ninety-four. The $E_{\mathrm{F}}$ stands for the Fermi level. The $\mathrm{C} 2 s$ and $\mathrm{C} 2 p$ represent the electric charge distributions of $2 s$ and $2 p$ orbits of the carbon atoms. The P2s and $\mathrm{P} 2 p$ represent the electric charge distributions of $2 s$ and $2 p$ orbits of the phosphorus atoms. The Lils $2 s$ represent the electric charge distributions of $1 s$ and $2 s$ orbits of the lithium atoms.

tively reduce the vacancies and the defects of the doped thin films, thus maintaining the integrity of the films. In the figures, the part of the conduction band near the Fermi level mainly comes from the contributions of the carbon atom's $2 p$ orbit, the phosphorus atom's $2 p$ orbit, a little of the carbon atom's $2 s$ orbit, the phosphorus atom's $2 s$ orbit and the lithium atom's $1 s$ and $2 s$ orbit. With different doping concentrations, the contributions of the carbon and phosphorus atom's $2 s$ orbit and the lithium atom's $s$ orbit are different. The part of the valence band near the Fermi level mainly comes from the contri-

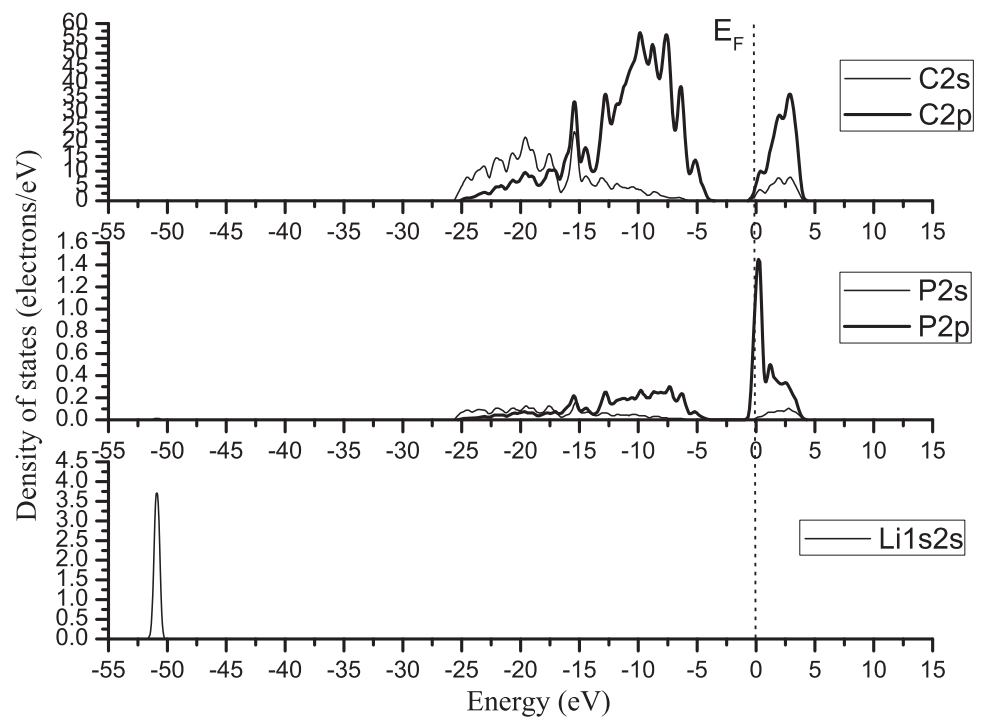

Figure 10. The partial density of states of the doped diamond where the number of Li atom is one, $\mathrm{P}$ atom's number is one and the C atom's number is one hundred and forty-two. The $E_{\mathrm{F}}$ stands for the Fermi level. The $\mathrm{C} 2 s$ and $\mathrm{C} 2 p$ represent the electric charge distributions of $2 s$ and $2 p$ orbits of the carbon atoms. The $\mathrm{P} 2 s$ and $\mathrm{P} 2 p$ represent the electric charge distributions of $2 s$ and $2 p$ orbits of the phosphorus atoms. The Li1 $s 2 s$ represent the electric charge distributions of $1 s$ and $2 s$ orbits of the lithium atoms. 


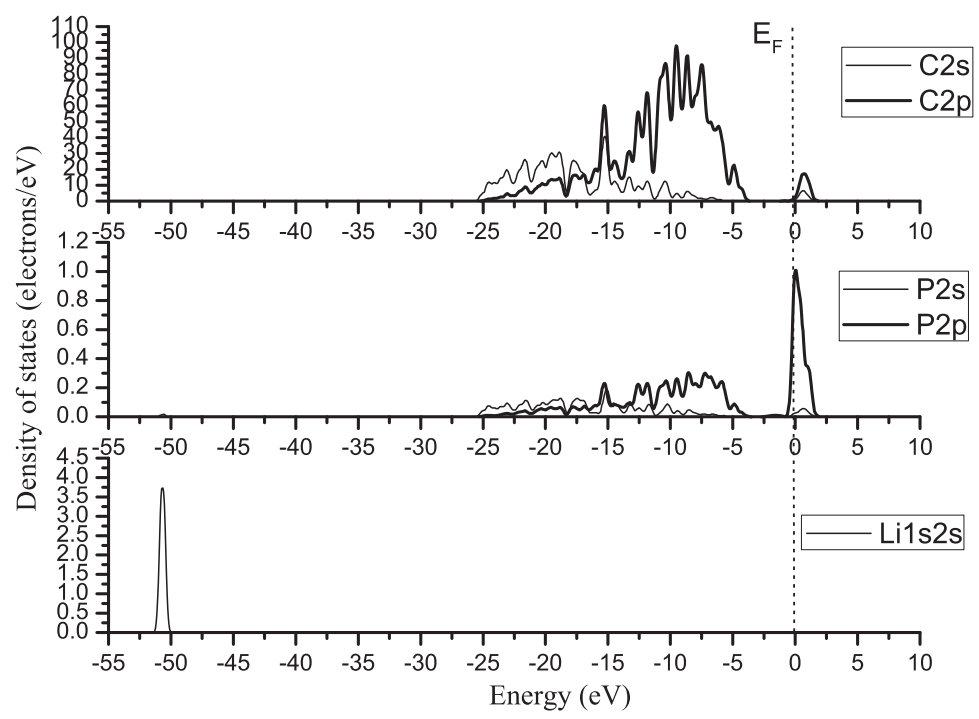

Figure 11. The partial density of states of the doped diamond where the number of $\mathrm{Li}$ atom is one, $\mathrm{P}$ atom's number is one and the C atom's number is two hundred and fourteen. The $E_{\mathrm{F}}$ stands for the Fermi level. The $\mathrm{C} 2 s$ and $\mathrm{C} 2 p$ represent the electric charge distributions of $2 s$ and $2 p$ orbits of the carbon atoms. The $\mathrm{P} 2 s$ and $\mathrm{P} 2 p$ represent the electric charge distributions of $2 s$ and $2 p$ orbits of the phosphorus atoms. The Lil $s 2 s$ represent the electric charge distributions of $1 s$ and $2 s$ orbits of the lithium atoms.

butions of the carbon atom's $2 p$ orbit and the phosphorus atom's $2 p$ orbit. As the doping concentration increases, the area of DOS of the valence band portion will increase. Therefore, when the Li-P atom-pair is incorporated into the diamond lattice, the covalent bond near the Li-P atom-pair will be destroyed. Thus, under the interactions among the outer electrons of the lithium atom, the phosphorus atom and the carbon atoms, the energy bands of the carbon atoms and the phosphorus atom will split near the Fermi level. The higher the concentration, the greater the energy band splits. And the split of the energy band near the Fermi level is propitious to improve the conductance properties of a semiconductor.

\subsection{Orbital charge distribution of the Li-P atoms and analysis of the bond length}

By the analysis of the Mulliken population, we can understand the orbital electron distributions of each atom in the Li-P co-doped diamond in detail, then to determine the bonding mechanism among different atoms. In the first line of table 1 the $S$ (or $P$ ) stands for the $s$ (or $p$ ) orbital charge after the atom combined with another atom, and the "Charge" stands for the charge an atom loses after it combined with another atom. The orbital charge distributions of the lithium atom and the phosphorus atom in the Li-P co-doped diamond are showed in table 1 In this table, both the atomic charges of the lithium atom and the phosphorus atom are positive, indicating that some charge of theirs has transferred to the vicinity of the carbon atoms. When the total atomic number of a cell is less than or equal to 32, the concentration of the impurity atoms is high. With the concentration decrease of impurity atoms, the $s$ orbital charge number of the lithium atom decreases from 1.99 to 1.17 , and the charge increases from 1.01 to 1.83 which the lithium atom loses after it combined with another atom. This indicates that when the doping concentration is high, then the higher is the doping concentration of lithium atom, the less is the charge contributing to the bonding of the lithium atom. While the total atomic number of a cell is more than 32 , the concentration of impurity atoms is low. The $s$ orbital charge number of the lithium atom and the charge which the lithium atom loses remain almost unchanged as the doping concentration changes. This indicates that when the doping concentration is low, the charge contributing to the bonding of the lithium atom almost does not change for different doping concentrations. As the doping concentration decreases, the $s$ orbital charge of the phosphorus atom first increases, then decreases and keeps an invariable value. As the doping concentration decreases, the $p$ orbital charge of the phosphorus atom decreases first, and then increases. Therefore, the total change of the $s$ and $p$ orbital charge of the phosphorus atom makes 
Table 1. The orbital charge distributions of the Li and P atoms in the Li-P co-doped diamond.

\begin{tabular}{|c|c|c|c|c|c|}
\hline \hline Number & atom & $S$ & $P$ & Total & Charge $(e)$ \\
\hline \hline \multirow{2}{*}{8} & $\mathrm{Li}$ & 1.99 & 0.00 & 1.99 & 1.01 \\
\cline { 2 - 6 } & $\mathrm{P}$ & 1.49 & 2.81 & 4.30 & 0.70 \\
\hline \multirow{2}{*}{16} & $\mathrm{Li}$ & 1.81 & 0.00 & 1.81 & 1.19 \\
\cline { 2 - 6 } & $\mathrm{P}$ & 1.54 & 2.67 & 4.21 & 0.79 \\
\hline \multirow{2}{*}{32} & $\mathrm{Li}$ & 1.17 & 0.00 & 1.17 & 1.83 \\
\cline { 2 - 6 } & $\mathrm{P}$ & 1.21 & 2.54 & 3.75 & 1.25 \\
\hline \multirow{2}{*}{64} & $\mathrm{Li}$ & 0.98 & 0.00 & 0.98 & 2.02 \\
\cline { 2 - 6 } & $\mathrm{P}$ & 1.21 & 2.63 & 3.84 & 1.16 \\
\hline \multirow{2}{*}{92} & $\mathrm{Li}$ & 1.13 & 0.00 & 1.13 & 1.87 \\
\cline { 2 - 6 } & $\mathrm{P}$ & 1.21 & 2.54 & 3.75 & 1.25 \\
\cline { 2 - 6 } & $\mathrm{Li}$ & 0.97 & 0.00 & 0.97 & 2.03 \\
\hline \multirow{2}{*}{144} & $\mathrm{P}$ & 1.21 & 2.67 & 3.88 & 1.12 \\
\cline { 2 - 6 } & $\mathrm{Li}$ & 0.96 & 0.00 & 0.96 & 2.04 \\
\hline \multirow{2}{*}{216} & $\mathrm{P}$ & 1.21 & 2.69 & 3.90 & 1.10 \\
\cline { 2 - 6 } & $\mathrm{Pi}$ & 0.96 & 0.00 & 0.96 & 2.04 \\
\hline \hline
\end{tabular}

the charge which the phosphorus loses after the bonding increases first, and then decreases. From the above mentioned, combined with the analysis of the DOS in section 2.1, we can find that the interactions of the outer electrons between the lithium atom, the phosphorus atom and the carbon atoms are very strong. When the doping concentration is high (or the total atomic number of a cell is less than or equal to 32), the doping concentration has a great effect on the $s$ orbital charge contributing to the bonding of the lithium and phosphorus atoms. When the doping concentration is low (or the total atomic number of the cell is more than 32), the doping concentration nearly does not affect the $s$ orbital charge contributing to the bonding of the lithium and phosphorus atoms, which keeps almost an invariable value. Moreover, no matter whether in the high doping or in the low doping diamond, the doping concentration also has a great effect on the $p$ orbital charge contributing to bonding of phosphorus atoms. The above results may provide some reference to doping experiments of a diamond.

When the lithium atom and phosphorus atom are incorporated into the diamond, the impurity atoms can form the $\mathrm{Li}-\mathrm{P}$ bond, the $\mathrm{Li}-\mathrm{C}$ bonds and the $\mathrm{P}-\mathrm{C}$ bonds. The bond lengths and the bond populations of the Li-P bond, the nearest neighbor Li-C bond and the nearest neighbor P-C bond are showed in table 2 As the table shows, the bond populations of the $\mathrm{Li}-\mathrm{C}$ atoms are all less than zero. When the doping concentrations of the impurity atoms are low (as the total atomic number of the crystal cell is more than 64), the bond population of the $\mathrm{Li}-\mathrm{C}$ atoms is equal to about -0.32 . This indicates that the $\mathrm{Li}-\mathrm{C}$ bond is anti-bonding, and as the doping concentration decreases, the anti-bonding states reach a stable value -0.32 . Except that the bond population of the Li-P atoms in the crystal cell whose total atomic number is 8 is positive, the other bond populations of the Li-P atoms are negative, and their values increase as the doping concentration increases. This indicates that the Li-P bond is also anti-bonding, but the constituents of the anti-bonding states decrease as the doping concentration increases. When the doping concentration reaches a certain value, the anti-bonding states of the Li-P atoms probably transfer into the bonding states (such as the situation of the crystal cell whose total atomic number is 8). The bond populations of the $\mathrm{P}-\mathrm{C}$ atoms are all positive, and in general their values increase as the doping concentration increases. This indicates that the $\mathrm{P}-\mathrm{C}$ bond is the bonding, and at the same time the constituents of the bonding states increase as the doping concentration increases. By the bond lengths shown in table 2 when the total atomic number of the crystal cell is 8 or 16 , the bond length of the Li-C bond is about $2.3 \AA$, and the average value of the Li-P atom's bond lengths is about $2.6 \AA$. When the total atomic number of the crystal cell is more than 32, the average value of the $\mathrm{Li}-\mathrm{C}$ atom's bond lengths is about 1.6 $\AA$, the average value of the Li-P atom's bond lengths is $2.0 \AA$. However, the bond lengths of the 
Table 2. The bond lengths and the bond population among the nearest neighbor $\mathrm{Li}-\mathrm{C}$ atoms, $\mathrm{Li}-\mathrm{P}$ atoms and the nearest neighbor P-C atoms in the Li-P co-doped diamond.

\begin{tabular}{|c|c|c|c|}
\hline Number & bond & population & length $(\AA)$ \\
\hline \multirow{3}{*}{8} & $\overline{\mathrm{Li}-\mathrm{C}}$ & -0.12 & 2.31932 \\
\hline & $\mathrm{Li}-\mathrm{P}$ & 0.12 & 2.55735 \\
\hline & $\mathrm{P}-\mathrm{C}$ & 0.71 & 1.75806 \\
\hline \multirow{3}{*}{16} & $\mathrm{Li}-\mathrm{C}$ & -0.04 & 2.34108 \\
\hline & $\mathrm{Li}-\mathrm{P}$ & -0.03 & 2.70780 \\
\hline & $\mathrm{P}-\mathrm{C}$ & 0.66 & 1.77178 \\
\hline \multirow{3}{*}{32} & $\mathrm{Li}-\mathrm{C}$ & -0.12 & 1.63001 \\
\hline & $\mathrm{Li}-\mathrm{P}$ & -0.26 & 2.09692 \\
\hline & $\mathrm{P}-\mathrm{C}$ & 0.66 & 1.74612 \\
\hline \multirow{3}{*}{64} & $\mathrm{Li}-\mathrm{C}$ & -0.32 & 1.63768 \\
\hline & $\mathrm{Li}-\mathrm{P}$ & -0.33 & 1.97483 \\
\hline & $\mathrm{P}-\mathrm{C}$ & 0.59 & 1.68708 \\
\hline \multirow{3}{*}{72} & $\mathrm{Li}-\mathrm{C}$ & -0.17 & 1.59965 \\
\hline & $\mathrm{Li}-\mathrm{P}$ & -0.29 & 2.02061 \\
\hline & $\mathrm{P}-\mathrm{C}$ & 0.64 & 1.72473 \\
\hline \multirow{3}{*}{96} & $\mathrm{Li}-\mathrm{C}$ & -0.32 & 1.62917 \\
\hline & $\mathrm{Li}-\mathrm{P}$ & -0.31 & 1.96159 \\
\hline & $\mathrm{P}-\mathrm{C}$ & 0.53 & 1.68730 \\
\hline \multirow{3}{*}{144} & $\mathrm{Li}-\mathrm{C}$ & -0.32 & 1.63096 \\
\hline & $\mathrm{Li}-\mathrm{P}$ & -0.32 & 1.94782 \\
\hline & $\mathrm{P}-\mathrm{C}$ & 0.53 & 1.68045 \\
\hline \multirow{3}{*}{216} & $\mathrm{Li}-\mathrm{C}$ & -0.32 & 1.63744 \\
\hline & $\mathrm{Li}-\mathrm{P}$ & -0.35 & 1.94659 \\
\hline & $\mathrm{P}-\mathrm{C}$ & 0.29 & 1.67908 \\
\hline
\end{tabular}

P-C atoms are almost uneffected by the doping concentration, and the average value of their bond lengths is about $1.7 \AA$. From the above analysis, the doping concentration has a great impact on the bond lengths of the Li-C bond and the Li-P bond, but has a little impact on the bond length of the P-C bond.

\section{Conclusion}

By the first principle calculation theory of the DFT, in this paper we calculate the electrical properties (such as the DOS and the orbit charge distributions and so on) of different doping concentrations' Li-P co-doped diamonds. First of all, as the Li-P atoms are incorporated into the diamond, this makes the Fermi level of the Li-P co-doped diamond move into the vicinity of the bottom of the conduction band and the conductance property of the Li-P co-doped diamond thin film has been greatly improved. When the concentration of the impurity atoms is low (the concentrations of the lithium atom or the phosphorus atom are less than $2.35 \times 10^{21} \mathrm{~cm}^{-3}$ ), the Li-P co-doped diamond thin film presents the characteristic of the semiconductors. When the concentration of the impurity atoms is high (the concentrations of the lithium atom or the phosphorus atom are more than $2.35 \times 10^{21} \mathrm{~cm}^{-3}$ ), the Li-P co-doped diamond thin film presents the characteristic of the conductors. Secondly, when the doping concentration is high, the $1 s$ and $2 s$ orbits of the lithium atom will have some contributions to the conduction band near the Fermi level, as well as may promote the splits of the phosphorus atom's and the carbon atom's $2 p$ orbits near the Fermi level. Thus, this is helpful to improve the electron conductivity of the Li-P co-doped diamond. The orbital charge distributions of the Li-P atoms also illustrate this phenomenon in detail. Finally, the incorporation of the lithium atoms into the doped diamond not only improves the electron conductiv- 
ity of semiconductors, but also may reduce the vacancies and defects of the doped diamond thin films. At the same time, the doping concentration of the impurity atoms also has a great impact on the bond lengths of the $\mathrm{Li}-\mathrm{C}$ atoms and the Li-P atoms. To sum up, the lithium atoms have an effect on the electron conductivity and the integrity of the diamond lattice in the Li-P co-doped diamond thin films.

\section{Acknowledgements}

This work was supported by the Natural Science Foundation of Fujian Province of China (A0220001).

\section{References}

1. Liao Z.H., Farrell J., J. Appl. Electrochem., 2009, 39, 1993; doi 10.1007/s10800-009-9909-Z

2. Luong J.H.T., Male K.B., Glennon J.D., Analyst, 2009, 134, 1965; doi 10.1039/b910206j

3. Andrade L.S., Rocha R.C., Cass Q.B., Fatibello O., Anal. Methods, 2010, 2, 402; doi 10.1039/b9ay00092e

4. Yuan J.J., Li H.D., Gao S.Y., Lin Y.H., Li H.Y., Chem. Commun., 2010, 46, 3119; doi 10.1039/c003172k

5. Sopik B., New J. Phys., 2009, 11, 103026; doi 10.1088/1367-2630/11/10/103026

6. Mandal S., Naud C., Williams O.A., Bustarret E., Omnes F., Rodiere P., Meunier T., Saminadayar L., Bauerle C., Nanotechnology, 2010, 21, 195303; doi 10.1088/0957-4484/21/19/195303

7. Oki N., Kagayama T., Shimizu K., Kawarada H., J. Phys. Conf. Ser., 2010, 215, 012143; doi $10.1088 / 1742-6596 / 215 / 1 / 012143$

8. Jones R., Lowther J.E., Goss J., Appl. Phys. Lett., 1996, 69, 2489; doi 10.1063/1.117715

9. Koide Y., Koizumi S., Kanda H., Suzuki M., Yoshida H., Sakuma N., Ono T., Sakai T., Appl. Phys. Lett., 2005, 86, 232105; doi $10.1063 / 1.1944896$

10. Kato H., Futako W., Yamasaki S., Okushi H., Diam. Relat. Mater., 2005, 14, 340; doi 10.1016/j.diamond.2004.11.032

11. Kato H., Yamasaki S., Okushi H., Diam. Relat. Mater., 2005, 14, 2007; doi 10.1016/j.diamond.2005.08.021.

12. Yamada T., Okano K., Yamaguchi H., Kato H., Shikata S., Nebel C.E., Appl. Phys. Lett., 2006, 88, 212114; doi $10.1063 / 1.2206552$

13. Pernot J., Koizumi S., Appl. Phys. Lett., 2008, 93, 052105; doi 10.1063/1.2969066

14. Cao G.Z., Driessen F.A.J.M., Bauhuis G.J., Giling L.J., Alkemade P.F.A., J. Appl. Phys., 1995, 78, 3125; doi $10.1063 / 1.359998$

15. Li R.B., Hu X.J., Shen H.S., He X.C., J. Mater. Sci., 2004, 39, 1135; doi 10.1023/B:JMSC.0000012963.04082.a1

16. Lee W.S., Yu J., Lee T.Y., J. Mater. Sci., 2005, 40, 5549; doi 10.1007/s10853-005-4548-1

17. Lombardi E.B., Mainwood A., Osuch K., Phys. Rev. B, 2007, 76, 155203; doi 10.1103/PhysRevB.76.155203

18. Iori F., Ossicini S., Physica E, 2009, 41, 939; doi 10.1016/j.physe.2008.08.010

19. Segall M.D., Lindan P.J.D, Probert M.J.J., J. Phys.: Condens. Matter, 2002, 14, 2717; doi 10.1088/0953-8984/14/11/301

20. Vanderbilt D., Phys. Rev. B, 1990, 41, 7892; doi 10.1103/PhysRevB.41.7892

21. Perdew J.P., Burke K., Ernzerhof M., Phys. Rev. Lett., 1996, 77, 3865; doi 10.1103/PhysRevLett.77.3865

22. Ching W.Y., Xu Y.N., Wong K.W., Phys. Rev. B, 1989, 40, 7684; doi 10.1103/PhysRevB.40.7684 


\title{
Першопринципні розрахунки літієво-фосфорного співлегованого алмазу
}

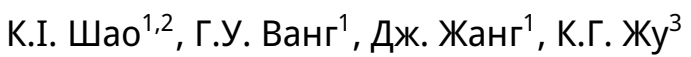 \\ 1 Лабораторія квантово-інформаційних технологій, Школа фізики та телекомунікаційних технологій, \\ Педагогічний університет Південного Китаю, Гуанчжоу 510006, Китай \\ 2 Фізичний факультет, Педагогічний університет Жангжоу, Жангжоу 363000, Китай \\ 3 Фізичний факультет, Бейханський університет, Пекін 100191, Китай
}

\begin{abstract}
Ми обчислюємо густину станів (DOS) і заселення Муллікена алмазу та співлегованих алмазів з різними концентраціями літію (Li) і фосфору (P) за допомогою методу функціоналу густини та аналізуємо випадки зв'язування тонких плівок Li-P співлегованого алмазу, а також впливи Li-P співлегування на провідність алмазу. Результати показують, що атоми Li-P можуть активізувати розщеплення енергетичної зони алмазу поблизу рівня Фермі, а отже покращити провідність електронів тонких плівок Li-P співлегованого алмазу, або ж навіть перетворити Li-P співлегований алмаз з напівпровідника у провідник. Проаналізовано вплив Li-P концентрації співлегування на орбітальний розподіл заряду, довжину зв'язку та заселеність зв'язку. Атом Li може активізувати розщеплення енергетичної зони поблизу рівня Фермі, а також може благотворно регулювати спотворення та розширення кристалічної гратки алмазу.
\end{abstract}

Ключові слова: Li-P співлегований алмаз, густина станів, рівень домішок, орбітальний заряд 